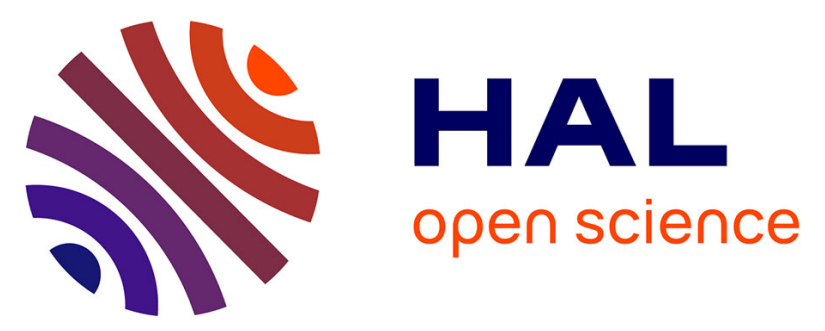

\title{
Recognition of white matter bundles using local and global streamline-based registration and clustering
}

Eleftherios Garyfallidis, Marc-Alexandre Côté, Francois Rheault, Jasmeen

Sidhu, Janice M Hau, Laurent Petit, David Fortin, Stephen Cunanne, Maxime

Descoteaux

\section{To cite this version:}

Eleftherios Garyfallidis, Marc-Alexandre Côté, Francois Rheault, Jasmeen Sidhu, Janice M Hau, et al.. Recognition of white matter bundles using local and global streamline-based registration and clustering. NeuroImage, 2018, 170, pp.283-295. 10.1016/j.neuroimage.2017.07.015 . hal-01622403

\section{HAL Id: hal-01622403 \\ https://hal.science/hal-01622403}

Submitted on 26 Jan 2021

HAL is a multi-disciplinary open access archive for the deposit and dissemination of scientific research documents, whether they are published or not. The documents may come from teaching and research institutions in France or abroad, or from public or private research centers.
L'archive ouverte pluridisciplinaire HAL, est destinée au dépôt et à la diffusion de documents scientifiques de niveau recherche, publiés ou non, émanant des établissements d'enseignement et de recherche français ou étrangers, des laboratoires publics ou privés. 


\title{
Recognition of white matter bundles using local and global streamline- based registration and clustering
}

\author{
Eleftherios Garyfallidis ${ }^{\mathrm{a}, *}$, Marc-Alexandre Côté ${ }^{\mathrm{b}}$, Francois Rheault ${ }^{\mathrm{b}}$, Jasmeen Sidhu ${ }^{\mathrm{b}}$, \\ Janice Hau ${ }^{c, d}$, Laurent Petit ${ }^{\mathrm{d}}$, David Fortin ${ }^{\mathrm{f}}$, Stephen Cunanne ${ }^{\mathrm{e}}$, Maxime Descoteaux ${ }^{\mathrm{b}}$ \\ ${ }^{a}$ Department of Intelligent Systems Engineering, School of Informatics and Computing, Indiana University, Bloomington, USA \\ b Sherbrooke Connectivity Imaging Lab (SCIL), Computer Science Department, Université de Sherbrooke, Sherbrooke, Canada \\ ${ }^{\mathrm{c}}$ Brain Development Imaging Lab (BDIL), Department of Psychology, San Diego State University, USA \\ ' Groupe d' Imagerie Neurofonctionnelle, Institut des Maladies Neurodégénératives, UMR5293, CNRS, CEA Université de Bordeaux, Bordeaux, France \\ e Research Center on Aging and Faculté de Médecine et des Sciences de la Santé, Université de Sherbrooke, Sherbrooke, Québec, Canada \\ ${ }^{\mathrm{f}}$ Division of Neurosurgery and Neuro-Oncology, Faculty of Medicine and Health Science, University of Sherbrooke, Sherbrooke, Québec, Canada
}

\section{A R T I C L E I N F O}

Article history:

Received 30 December 2016

Received in revised form

6 June 2017

Accepted 10 July 2017

Available online 13 July 2017

Keywords:

Diffusion MRI

Fiber tracking

Streamlines

Bundles

Fascicles

Tracts

Recognition

Extraction

Clustering

Virtual dissection

\begin{abstract}
A B S T R A C T
Virtual dissection of diffusion MRI tractograms is cumbersome and needs extensive knowledge of white matter anatomy. This virtual dissection often requires several inclusion and exclusion regions-of-interest that make it a process that is very hard to reproduce across experts. Having automated tools that can extract white matter bundles for tract-based studies of large numbers of people is of great interest for neuroscience and neurosurgical planning. The purpose of our proposed method, named RecoBundles, is to segment white matter bundles and make virtual dissection easier to perform. This can help explore large tractograms from multiple persons directly in their native space. RecoBundles leverages latest state-of-the-art streamline-based registration and clustering to recognize and extract bundles using prior bundle models. RecoBundles uses bundle models as shape priors for detecting similar streamlines and bundles in tractograms. RecoBundles is $100 \%$ streamline-based, is efficient to work with millions of streamlines and, most importantly, is robust and adaptive to incomplete data and bundles with missing components. It is also robust to pathological brains with tumors and deformations. We evaluated our results using multiple bundles and showed that RecoBundles is in good agreement with the neuroanatomical experts and generally produced more dense bundles. Across all the different experiments reported in this paper, RecoBundles was able to identify the core parts of the bundles, independently from tractography type (deterministic or probabilistic) or size. Thus, RecoBundles can be a valuable method for exploring tractograms and facilitating tractometry studies.
\end{abstract}

(c) 2017 Elsevier Inc. All rights reserved.

\section{Introduction}

Current tractography algorithms produce a large number of streamlines (up to millions) that form a tractogram. Recently, it has been reported that tractography can generate a great number of invalid streamlines and white matter bundles (Côté et al., 2013; Maier-Hein et al., 2016; Jones et al., 2013; Thomas et al., 2014), which can create biases in tractometry analysis (Dayan et al., 2016; Bells et al., 2011; Yeatman et al., 2012; O'Donnell et al., 2009; Renauld et al., 2016) and connectivity analysis (Hagmann et al., 2008). A partial solution to this problem is to inject strong anatomical priors into the tractography and track-dissection processes (Girard et al., 2014; Chamberland et al., 2017). The term virtual dissection (Catani et al., 2002a) is often used when an interactive visualization tool (Wang et al., 2007a; Chamberland et al., 2014) is used to segment a bundle of interest while removing all streamlines and bundles that do not correspond to the anatomical definition of this bundle. For this reason, many groups still manually segment hundreds of bundles using visual tools (Chamberland et al., 2014) with inclusion/exclusion regions-of-interests (ROIs) (Wang et al., 2007b). This manual process is very time consuming, is strongly user-dependent and adds biases to tract-based analyses. It can take hundreds of hours for trainees and neuroanatomical experts to manually segment bundles over multiple participants. There is thus an important need for automatic tools that can facilitate the process and provide new databases of white matter bundles.

We can divide the existing automated tools into two categories: a)

\footnotetext{
* Corresponding author.

E-mail address: elef@indiana.edu (E. Garyfallidis).
} 
connectivity-based and b) streamline-based. In (a), the streamlines of the tractograms are filtered in relation to their connection to specific ROIs, often pre-defined in a template. These approaches require image-based multi-modal nonlinear registration, for example by registering a $\mathrm{T} 1$ to diffusion data (or the opposite) so that the streamlines and the ROIs are in the same space. In (b), an atlas of known bundles is given, and the streamlines of the atlas are used directly to perform the segmentations of the given tractograms.

Most published methods belong in category (a). For example, one of the most recent methods was introduced by Wassermann et al. (2016) where the authors created rules describing how known anatomical bundles connect different regions of the brain (parcellated using Freesurfer), and then used those rules to extract bundles from tractograms. Another method which is based on Freesurfer parcellations is Tracula (Yendiki et al., 2011; Siless et al., 2016), which also requires registration of an individual $\mathrm{T} 1$ to a common template. A similar approach had been proposed earlier by Zhang et al. (2010) and later by Tunç et al. (2014). These approaches all need to have multiple modalities in a common space (Yeatman et al., 2012; Jin et al., 2014) using image-based registration, a possible parcellation, and filtering of streamlines connecting different ROIs. Obtaining such a perfect alignment between streamlines and ROIs can be a difficult task especially when processing pathological brains. The present paper belongs in category (b). Here, most of the existing approaches are quite recent (O'Donnell and Westin, 2007; Guevara et al., 2012; Garyfallidis et al., 2015a; O'Donnell et al., 2017) and do need some form of initial registration (Olivetti et al., 2016; Durrleman et al., 2011). However, they mostly use distances between streamlines and clustering of streamlines to simplify the segmentation process. Furthermore, multiple bundle atlases can be used simultaneously to increase the accuracy of segmentation as shown by Yoo et al. (2015) and Labra et al. (2013, 2016).

In our novel approach, the need for fast clustering and simplification of large tractograms using streamline distances is addressed using QuickBundles (Garyfallidis et al., 2012) and the need for fast linear registration of streamlines is addressed using Streamline-based Linear Registration (SLR) by Garyfallidis et al. (2015b). We show how by combining these two methods at a global and local level we can create a powerful streamline-based segmentation method. The new method is called RecoBundles, which stands for recognition of bundles. RecoBundles is $100 \%$ streamline-based and is efficient to work with millions of streamlines. Moreover, we show that RecoBundles is robust and adaptive to incomplete data and bundles with missing components. It is also robust to pathological brains with tumors which deform or reduce the size of bundles. We quantitatively evaluated our results in 4 bundles manually dissected by 2 experts in 60 individuals and showed that RecoBundles is in overall good agreement with experts having high sensitivity and specificity. We also qualitatively show that RecoBundles discovers the core parts of the bundles and, overall, produces more complete (dense) bundles. The amount of filling can be adjusted by a free parameter $(\lambda)$ which is an advantageous feature for neuroanatomists wanting to explore the extent of specific tracts.

RecoBundles facilitates the testing of hypotheses about the existence of different bundles and running them across many participants. It opens novel perspectives for bundle-extraction for neurosurgical planning and tractometry. The algorithmic aspects of RecoBundles are explained in detail in Section 2 and the method is validated with simulations in Section 3.1. Furthermore, we show quantitative results with real datasets in Section 3.2.1 and qualitative results with healthy and patient datasets in Sections 3.2.2 and 3.3 .

\section{Methods}

\subsection{Intuition}

The term bundle recognition is inspired from the field of computer vision where object recognition describes the task of finding and identifying objects in an image or video sequence. Humans recognize a multitude of objects in images with little effort, despite the fact that the image of the objects may vary in size and scale or rotation. Similarly, in the field of neuroimaging, we want to identify bundles in tractograms which have differences in orientation, size and shape and which may be partly incomplete, based on shape priors from pre-segmented bundles, all with minimum effort, e.g. given only a single example bundle. This is different from atlas-based bundle extraction where one gives as input an entire set of bundles from an atlas as in Guevara et al. (2012); Labra et al. (2016). Here, given a certain number of streamline-based 'bundle models' generated from manual virtual dissection or semi-automatic segmentation of the whole brain tractogram, RecoBundles will automatically recognize and extract streamlines that have similar shapes to these models. For example, as seen in Fig. 1, one segments the left corticospinal tract (CST) of a single healthy individual based on its whole brain full tractogram in native space, as seen in $S_{1}$ of Fig. 1 . Then, RecoBundles can identify the left CST ( $S_{2}$ of Fig. 1 ) of another individual in its own native space as well using the healthy control CST as a shape prior for the bundle recognition. This individual is a patient with a large glioblastoma in the left hemisphere.

\subsection{Methodological details}

We denote a single streamline with $s$ and a set of streamlines with $S$. A streamline $s$ is an ordered sequence of line segments connecting 3D vector points $\mathbf{x}_{k} \in \mathbb{R}^{3}$ with $k \in[1, K]$, where $K$ is the total number of points of streamline $s$. A visual diagram of RecoBundles is shown in Fig. 2 to ease the understanding of the details of technique.

The inputs of our method are a) the whole brain tractogram of

\section{Recognition of CST with tumor using a healthy CS1}

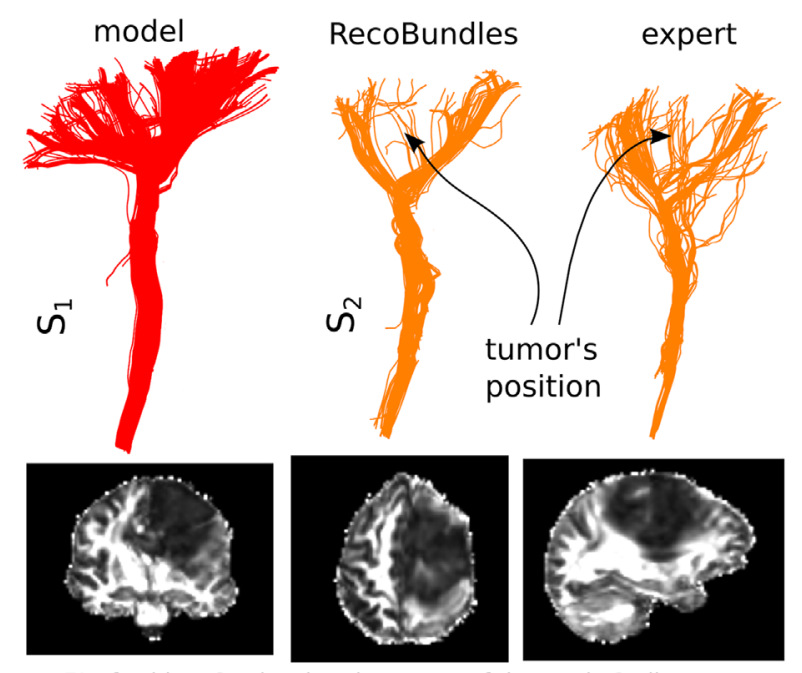

FA of subject $S_{2}$ showing the extent of the grade 3 glioma tumor

Fig. 1. We see the recognition of the CST (upper-center) in a brain with a large glioma grade 3 tumor. Notice how the tumor had affected the CST and how RecoBundles was able to identify it comparably to the expert's segmentation (upperright). The expert needed to add information from an fMRI finger tapping experiment to segment this bundle while RecoBundles identified it using a single healthy CST from a different individual (upper-left). At the lower panel we show the extent of the tumor's damage using FA slices. 
the participant $S_{A}$ from whom we want to recognize bundles, b) the whole brain streamlines $S_{M}$ from which we obtained manually segmented model bundles. For clarity, we divide the method in two phases. Phase A provides an initial rough streamline-based registration (usually affine), where we register to $S_{M}$. The affine registration is performed using the Streamline-based Linear Registration (SLR) method described by Garyfallidis et al. (2015b). Phase $B$ provides the recognition and is the main contribution of this paper. It is in this phase that we use a model bundle $B_{M} \subset S_{M}$ e.g., the left cingulum, and the goal is to recognize the same bundle in $S_{A}$.

To deal with large tractograms both phases use QuickBundles clustering of streamlines (see Garyfallidis et al., 2012). Also, we can accelerate many of the computations by analyzing the centroids of the clusters rather than the individual streamlines. The same streamline distance is used for clustering, registration and pruning. This is the Minimum average Direct-Flip (MDF) distance. The MDF is metric distance in the space of resampled streamlines as shown in Garyfallidis et al. (2012). Working with a distance metric has the advantage of simplifying many of the computations.

\subsubsection{Phase A: affine registration using global SLR}

The first phase starts with $S_{A}$ and $S_{M}$ being clustered using QuickBundles (see Fig. 2a-c). Then the centroids, and $C_{M}$, respectively are registered using SLR. In Fig. 2d using blue/purple color, the difference before and after registration of the centroids is shown. Only the centroids of the largest clusters are used because all clusters with less than $c$ streamlines are removed ( $c=50$ everywhere in the document). Only streamlines with lengths between 50 and $250 \mathrm{~mm}$ are used for the clustering. To further facilitate the process we only use the same number $R$ of randomly selected streamlines from $S_{A}$ and $S_{M}$ ( $R=50,000$ streamlines). After the SLR has finished, we apply the transformation to the initial streamlines $S_{A}$ (nothing was removed, i.e. the whole tractogram) such that $S_{A}$ is linearly registered in the space of $S_{M}$ (see Fig. 2e). We denote the transformed $S_{A}$ as $S_{A}{ }^{\prime}$. Once $S_{A}{ }^{\prime}$ is roughly registered with $S_{M}$ with affine registration, we can move to the second phase of the proposed method.

\subsubsection{Phase B: bundle recognition using local SLR}

The second phase starts with a clustering of $S_{A}{ }^{\prime}$ using QuickBundles. Now, QuickBundles is applied on the full dataset and new centroids are obtained (see Fig. 2e-f). The distance threshold used in QuickBundles is denoted $\theta_{S}$ (default value $15 \mathrm{~mm}$ ).

The model bundle, $B_{M}$, is also clustered but with a smaller distance threshold $\theta_{B}=\theta_{S} / 3$ (default value $5 \mathrm{~mm}$ ). Then, a far pruning operation is performed to remove all streamlines from $S_{A}{ }^{\prime}$ that are further than MDF distance $\Lambda$ (default value $20 \mathrm{~mm}$ ) from any centroid of $B_{M}$. This far pruning operation removes bundles with very different shapes or from a different hemisphere. It also allows us to focus on a smaller amount of streamlines than the initial $S_{A}{ }^{\prime}$. $S_{A}{ }^{\prime}$ after far pruning is shown in Fig. $2 \mathrm{~h}$. Let's denote this neighborhood of the model bundle as $N_{A} \subset S_{A}^{\prime}$ where $N_{A}$ stands for the neighborhood of $B_{M}$ in the target $S_{A}$ space. Next, $N_{A}$ is registered to $B_{M}$ using (SLR) and the transformation matrix from the registration is applied to $N_{A}$. We denote the transformed $N_{A}$ as $N_{A}{ }^{\prime}$. This step has performed not only a registration but also a matching operation because the SLR is robust to incomplete and missing data as shown in Garyfallidis et al. (2015b). Therefore, the SLR will register only the parts of $N_{A}$ that look like the shape of $B_{M}$ and ignore the rest. After having $N_{A}{ }^{\prime}$ and $B_{M}$ matched (see Fig. $2 \mathrm{i}$ ), we can remove all streamlines (local pruning) from $N_{A}{ }^{\prime}$ that are farther than a threshold $\lambda$ (usually set between 5 and $8 \mathrm{~mm}$ ) from the centroids of $B_{M}$. Changing $\lambda$ allows the users to shrink or expand the final result (see section 2.2.3). The final extracted bundle is shown in Fig. 2 j. We denote this bundle as $B_{A}$. We can now clearly see that the recognized bundle $B_{A}$ in Fig. $2 \mathrm{j}$ is very similar to the model bundle $B_{M}$ in Fig. $2 \mathrm{~g}$.

\subsubsection{Pruning operation}

Both far and local pruning operations work in an efficient way by computing a distance matrix of the centroids rather than using all the streamlines. For example, for the far pruning we calculate the distance matrix between the centroids of $S_{A}{ }^{\prime}$ and the centroids of $B_{M}$. We keep all the streamlines from $S_{A}{ }^{\prime}$ that belong to clusters with centroids which have a MDF distance less than $\Lambda$ from at least one of the centroids of $B_{M}$. The same idea is used for local pruning but between $N_{A}{ }^{\prime}$ and $B_{M}$. See Fig. 3 for an example of the effect of $\lambda$. Here the model bundle is an inferior longitudinal fasciculus (ILF) that had 337 streamlines and the expert segmented 1926 streamlines. RecoBundles generated 766 streamlines at $5 \mathrm{~mm}$ and 2033 at $7 \mathrm{~mm}$ which is closer to the amount of streamlines generated by the expert.

\subsubsection{Cost function}

The cost function used for the two registrations used in Phase A and Phase B was the same used in Garyfallidis et al. (2015b). Given two sets of streamlines, we denote $S_{a}=\left\{s_{1}^{a}, \ldots, s_{A}^{a}\right\}$ and $S_{b}=\left\{s_{1}^{b}, \ldots, s_{B}^{b}\right\}$, where $A$ and $B$ are the total number of streamlines in each set respectively. We want to minimize a cost function so that we can align the two sets together. For this purpose, we use the Bundle-based Minimum Distance (BMD), which is defined as:

$\operatorname{BMD}\left(S_{a}, S_{b}\right)=\frac{1}{4}\left(\frac{1}{A} \sum_{i=1}^{A} \min _{j} D_{i j}+\frac{1}{B} \sum_{j=1}^{B} \min _{i} D_{i j}\right)^{2}$

where $D$ is the rectangular matrix given by all pairwise MDF streamline distances (Garyfallidis et al., 2012). Therefore, every element of matrix $D$ is equal to $D_{i j}=\operatorname{MDF}\left(s_{i}^{a}, s_{j}^{b}\right)$. Notice, how in eq. (1), the most similar streamlines from one streamline set to the other are weighted more by averaging the minimum values of the rows and columns of matrix $D$.

During Phase $A$, we minimize the cost function between the centroids of $S_{A}$ and $S_{M}$. During Phase B we do something different. We minimize the cost function by randomly selecting a specific number of streamlines from $N_{A}$ and the same from $B_{M}$. Also, another difference is that in Phase $A$ we perform affine registration by default but in Phase $B$ we use rigid registration by default. Nonetheless, the option of changing the type of registration in the different phases is available to the user.

\section{Experiments and results}

\subsection{Simulations}

In this experiment, we studied the behavior of RecoBundles in specific and simulated conditions. We wanted to validate RecoBundles for recognizing bundles with deformations. For this purpose, we combined all bundles of the ISMRM 2015 challenge (details in tractometer.org ${ }^{1}$ and description in Appendix B) to form a full tractogram, then took one of the bundles, deformed it and replaced it in the initial tractogram. So, now we have two tractograms. $S_{M}$ is the initial tractogram including all bundles without defects and $S_{A}$ contains all initial bundles except one which was replaced by its deformed version. The idea here was to use the non-deformed bundle as the model and try to identify the deformed bundle using RecoBundles. Ideally, the indices of the two bundles in tractograms and $S_{M}$ should be identical if the

\footnotetext{
${ }^{1}$ http://tractometer.org/ismrm_2015_challenge.
} 


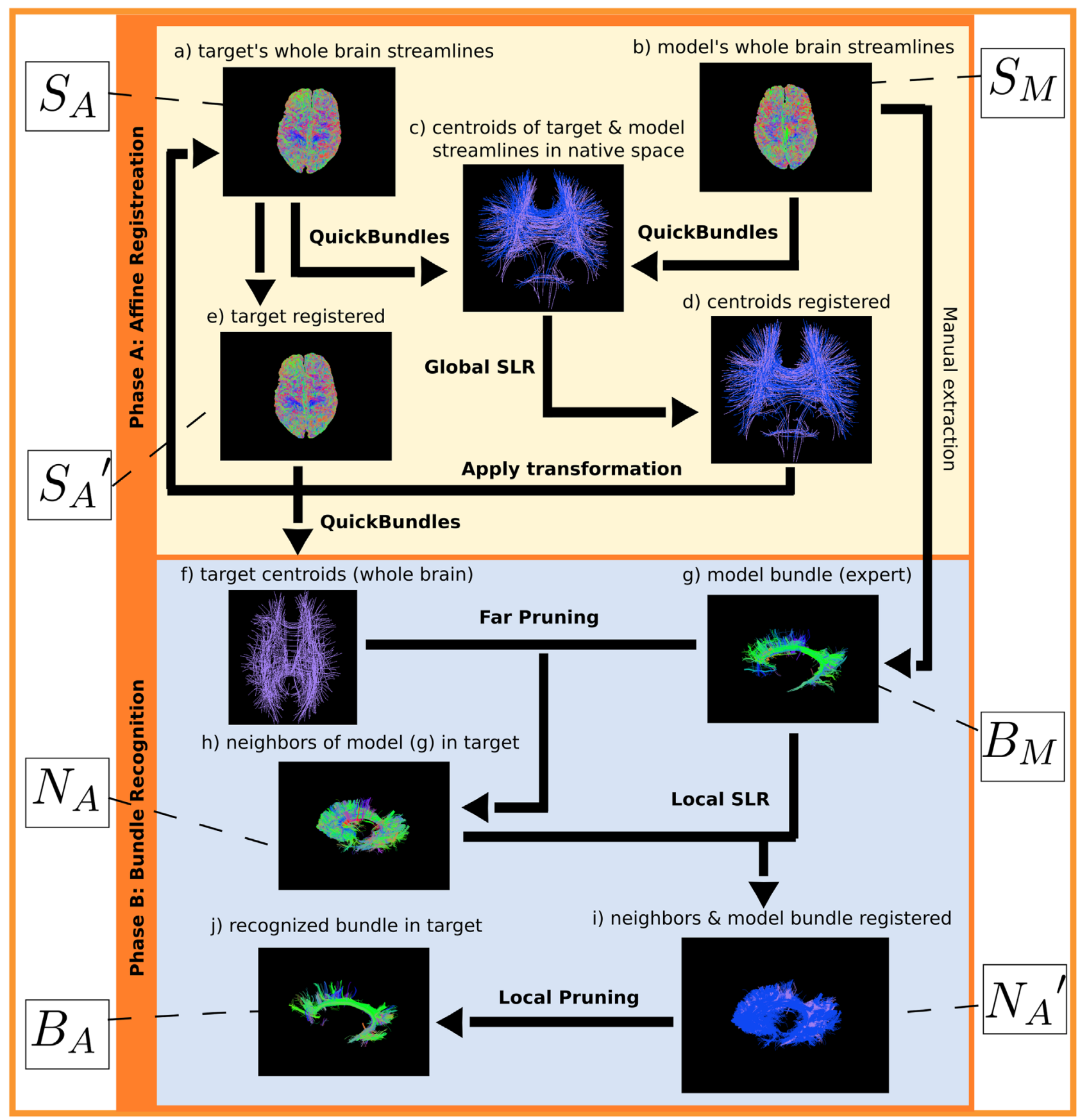

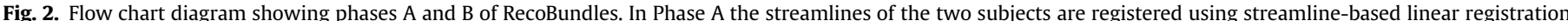

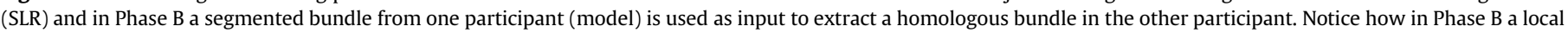
SLR is also executed. This SLR is responsible for matching the bundles.

recognition was successful.

At the right side of Fig. 4 we see an example of the simulation of the defect. For example, in Fig. 4a, a bundle from the ISMRM 2015 white matter challenge is selected (here the anterior commissure - CA) which is then deformed with different defects that can take place in any part of the bundle as shown in Fig. $4 \mathrm{~b}$ and c. The defect is simulated by moving the points $\mathbf{p}$ of the streamlines according to their distance from a center c. The center can be anywhere in the bundle, and its position is selected using an index $i \sim U(1,|A|)$ where $|A|$ is the total number of streamlines, and $j \sim U\left(1,\left|S_{i}\right|\right)$ where $\left|S_{i}\right|$ is the number of points for the streamline $i$. That means, $\mathbf{p} \rightarrow S(i, j) \in \mathbb{R}^{3}$. $\mathbf{p}$ is being updated with the following equation to create the defect (simulated anomaly). $\mathbf{p}=\mathbf{p}+r \frac{\mathbf{v}}{\|\mathbf{v}\|_{2}} \exp \left(-\frac{\|\mathbf{v}\|_{2}^{2}}{2 \sigma^{2}}\right)$

where $\mathbf{v}=\mathbf{p}-\mathbf{c}, r \sim U(0,5)$ is the strength of the defect, $\sigma \sim U(1,6)$ is the standard deviation of the Gaussian in Eq. (2). The behavior of this equation is shown in Fig. $4 \mathrm{~b}$ and c. The maximum displacement area has diameter $10 \mathrm{~mm}$.

Now, with this experiment, we can validate RecoBundles by looking at the indices of the streamlines of the recognized bundle in contrast to the indices of the streamlines of the initial model bundles. The standard method here is to look at the Jaccard index (J) of their labels defined as $\frac{B_{M} \cap B_{A}}{B_{M} \cup B_{A}}$ where $B_{M}$ is the set of indices of the initial bundle and $B_{A}$ is the set of indices of the recognized 


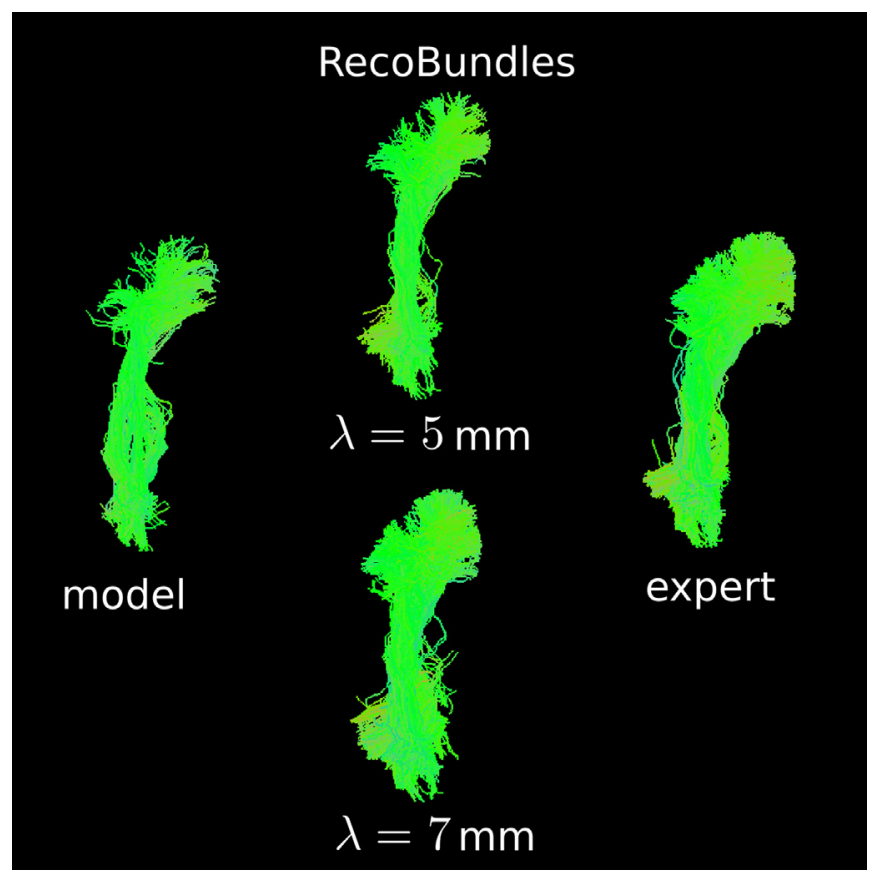

Fig. 3. Showing the change in RecoBundles' vs expert's segmentation after modifying the pruning threshold $\lambda$. Notice how by increasing/reducing $\lambda$ we can expand/ shrink the final bundle (middle column).

bundle.

We sampled the parameters $\mathbf{c}, r, \sigma, \mathbf{t} 50$ times for each bundle and used the same RecoBundles parameters for all. The parameters used were cluster_thr $=15$, reduction_thr $=15$, transformation $=$ rigid, pruning_thr $=5$ (all units are in $\mathrm{mm}$ ).

As we can see in the boxplot diagram, RecoBundles performed nearly always close to perfect match $(\mathrm{J}=1)$ and always performed higher than 0.75 , which is a very high Jaccard index (see Avants et al. (2011)). This experiment showed that RecoBundles is capable of correctly identifying bundles with deformations and therefore provided a positive indication for moving ahead with the detection of bundles in healthy brains and brains with tumors.

\subsection{Comparisons against expert anatomists}

\subsubsection{Quantitative evaluation in 60 subjects}

In this experiment, we applied RecoBundles to identify the left and right inferior fronto-occipital fasciculi (IFOF) and uncinate fasciculi (UNC) ( 4 bundles in total) in 60 different subjects. The tracking algorithm used with these datasets was FACT (Mori et al., 1999) which is known to generate many incomplete streamlines because it is based on a FA threshold and cannot resolve crossing areas. For the full description of the dataset and tracking generation procedure see Appendix C. The main reason for selecting this dataset for validation was that 2 independent neuroanatomists manually segmented these 4 bundles in all 60 different subjects, giving us the opportunity to quantitatively measure how RecoBundles compares to expert segmentations and see how it performs in noisy and realistic data with good sample size. This dataset also has the advantage that it contains bundles that were shorter and curved (e.g. UNC) and bundles that were longer and straighter (e.g. IFOF) and their lateral counterparts. Virtual (manual) segmentation is a long and laborious task. The expert neuroanatomists spent many days segmenting those 4 bundles in 60 subjects using Trackvis, as described in Hau et al. (2016).

In Fig. 5, we see how RecoBundles was able to recognize the UNC comparably to an expert in four different subjects. RecoBundles performed similarly in 60 subjects in total. The model bundle used was another uncinate bundle from a different participant.

To validate RecoBundles quantitatively and confirm the visual inspection shown in Fig. 5, we used cross validation. From the 60 subjects, one was randomly selected. This became the model tractogram $S_{M}$. For this participant we used the bundle segmented by a neuroanatomist as the model bundle $B_{M}$. RecoBundles used this model bundle to extract bundles in the other 59 subjects' tractograms. We repeated this experiment 20 times, each time randomly selecting a different participant (without replacement) to be the one used as the model tractogram. Therefore, in this experiment RecoBundles was executed $59 \times 20 \times 4=4720$ times in total. To measure the performance of the recognition we used the standard and well established techniques from binary classification both using directly the indices of the streamlines (see Table 1) or looking at their spatial extent (see Table 2). In both ss, we assumed that the experts' segmentations were the gold standard. The numbers of subjects used as models where 20 from a pool of 60 subjects. The parameters used were reduction_thr 20 , clust_thr 15, matrix $400 \times 400$ and pruning_thr 8 . These were the same for all the bundles.

To help the reader, the following abbreviations are used: true positives $(T P)$, true negatives $(T N)$, false positives $(F P)$ and false negatives $(F N)$. In Table 1 we see that the sensitivity $(T P /(T P+F N))$ is relatively high (between 0.68 and 0.92 on average), indicating that few streamlines that were detected by the expert, were missed by RecoBundles because $F N$ is small in comparison to TP. The specificity $(T N /(T N+F N))$ was very high as expected as $F N$ is much lower than the number of $T N$. $T N$ is expected to be high as the tractogram is much larger (approximately 150,000 streamlines per participant) than the size of a specific bundle (maximum around 1500 streamlines). Now, the accuracy $(T P+T N) /$ $(T P+T N+F P+F N)$ was also very high for all bundles shown that the total number of false predictions $(F P+F N)$ was much smaller than the number of true predictions $(T P+T N)$. So far, all these metrics were expected. However, precision $T P /(T P+F P)$ and the Jaccard index $(T P /(T P+F N+F P))$ were around $20-30 \%$ this is because the FP were greater than TP. This means also that RecoBundles is generating more dense bundles than the experts as visually the bundles look similar, but the precision is low. As shown in the last two columns of Table 1 where the size of recognized bundles (Rec_Size) is 2-3 times higher than the size of the expert bundles (Exp_Size). The current experiment is very precise because it uses the indices of the bundles on the tractogram to calculate the binary classification metrics.

We calculated the sensitivity and Jaccard index taking into account the spatial extent (number of voxels traversed by streamlines). This is presented in Table 2. Notice that both measures are increasing in comparison to Table 1 . This indicates that the bundles are denser. This can also be seen qualitatively in Fig. 5 . We can also confirm this by looking at the metric called bundle adjacency introduced in Garyfallidis et al. (2012). This normalized metric shows which percentage of streamlines from the recognized bundles has neighbors from the set of expert bundles or vice verca. To detect if a streamline has a neighbor from the other set we use the MDF distance and a low distance threshold (here at $2 \mathrm{~mm}$ ). In Table 1 we can see that on average $53-68 \%$ of streamlines have close neighbors. This is another quantitative confirmation that the recognition was largely successful. Note that nothing was done to pre-process the model bundles in any way. The model bundles were randomly picked as the experts segmented them according to their criteria without removing short streamlines or any other technique which could potentially make the recognition process easier. These are all rough unprocessed models. 


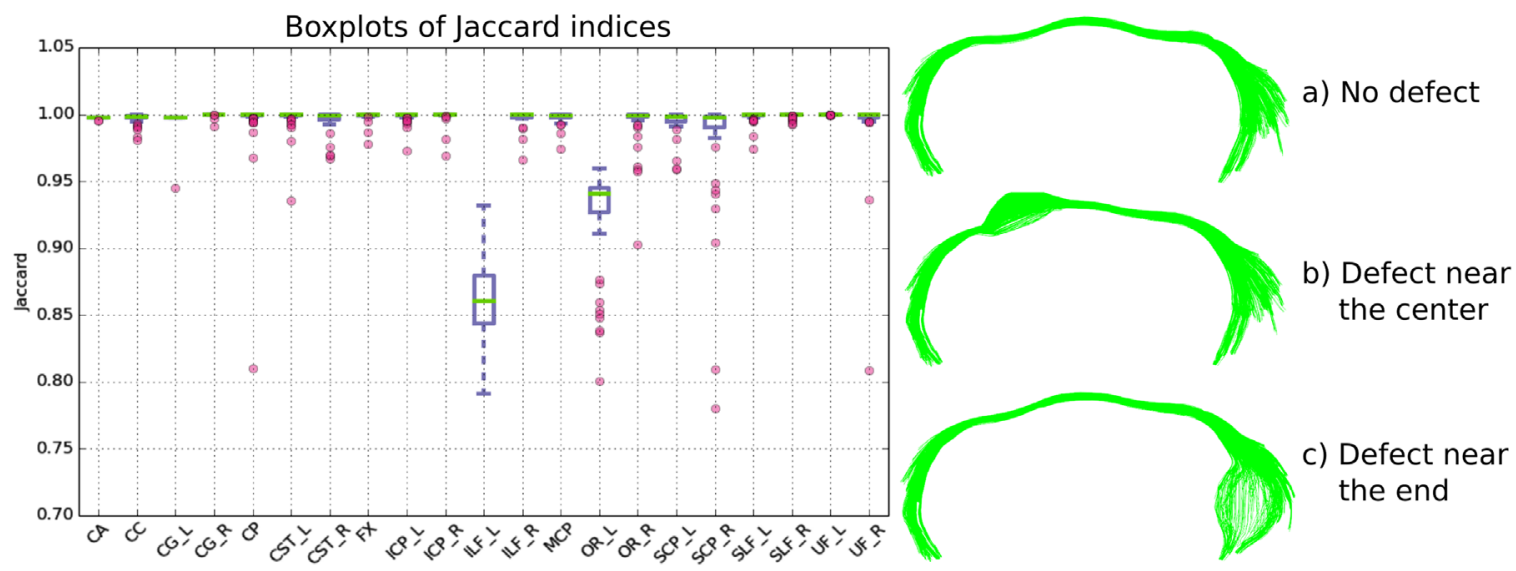

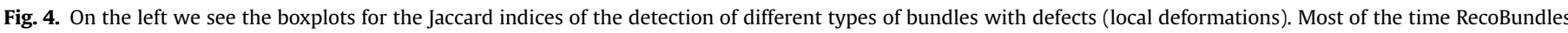

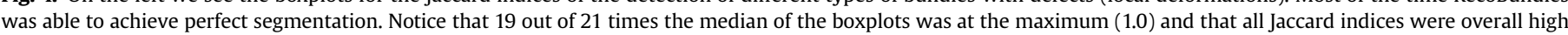

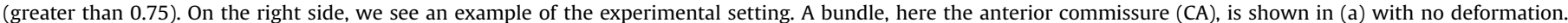

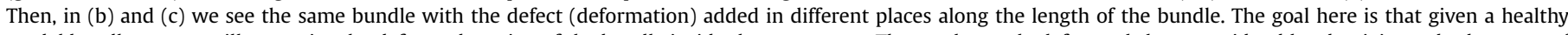

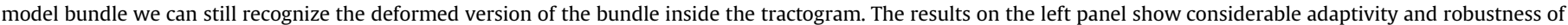
RecoBundles in local defects.

\section{Recognizing uncinate}

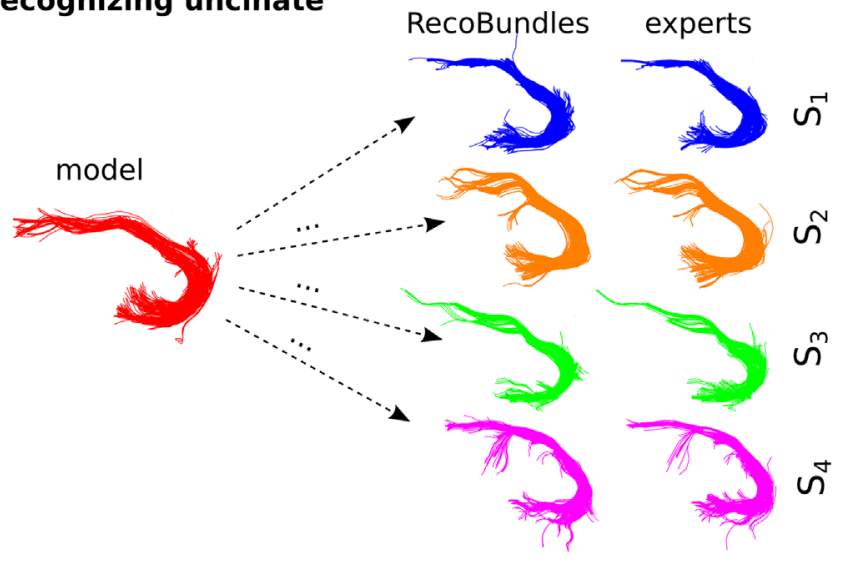

Fig. 5. At the left, we see the left uncinate model bundle with red segmented by an expert and used as input to RecoBundles to automatically segment the same type of bundle from 4 other tractograms $\left(S_{1}-S_{4}\right)$. On the right side, we see the expert segmentations for this type of bundle in the same four tractograms. Notice how similar the automatic segmentations are using RecoBundles compared to the expert segmentations.

\subsubsection{Qualitative evaluation}

Here we describe the qualitative evaluation of two healthy subjects using probabilistic tractography. In this experiment we used two public datasets from the Human Connectome Project (HCP). Participants 210,011 and 152,831. The tractogram of participant 210,011 had 517,633 streamlines (1.79 GBytes) and participant 152,831 had 494,521 streamlines (1.68 Gbytes). These datasets were of higher resolution from the ones used in the previous sections and are generated with probabilistic tractography which creates more fluctuating streamlines. MAPMRI (Özarslan et al., 2013) was used for reconstruction, and probabilistic tracking with particle filtering (Girard et al., 2014) was used for generating the tractograms. A different and independent anatomist from the anatomist used in the previous sections segmented 8 bundles in both subjects. Those were the left and right cingulum (CG), left and right inferior fronto-occipital fasciculi (IFOF), left and right optic radiation, the splenium of corpus callosum (SPL) and the middle cerebellar peduncle (MCP). More details about the datasets and expert segmentation strategies are given in Appendix D.

The expert neuroanatomist was asked to segment the first participant's (210011) tractogram $S_{M}$. The bundles of this participant $\left(B_{M}^{i}\right)$ were used as model bundles for RecoBundles. The expert was asked then to segment the bundles of the tractogram $S_{E}$ of participant 152,831 (the subscript $E$ stands for expert). We denote the segmented bundles as $B_{E}^{i}$. Finally, the expert bundles of $B_{E}^{i}$ were qualitatively compared with the output of RecoBundles applied on the same participant using as model bundles the $B_{M}{ }^{i}$. We denote the RecoBundles bundles as $B_{A}^{i}$. Notice here that $i$ is the index of one of the 8 bundles. The results are shown in Fig. 6 .

In the first row, we see that the cingulum bundle is well recognized by RecoBundles. Although the model bundle does not have as many streamlines to the frontal lobe as the one identified by the expert, RecoBundles is able to detect more anterior streamlines than the expert for CG_R. This is a straightforward example showing how adaptive RecoBundles is and can produce useful results when using incomplete models. In summary, the model bundle had 4064 streamlines, the RecoBundles bundle had 7499 streamlines and the expert bundle had only 4670 streamlines. In the second row we see a similar outcome with RecoBundles detecting a more dense bundle than the expert for CG_L although the model is not as dense (model 4117, RecoBundles 7224 and expert 2425 streamlines).

All the parameters used for RecoBundles are the same for all the bundles of this experiment (reduction_thr $20 \mathrm{~mm}$, clust_thr $15 \mathrm{~mm}$, matrix $400 \times 400$ and pruning_thr $5 \mathrm{~mm}$ ). In the third and forth rows we see a different behavior with RecoBundles not detecting such a dense IFOF as the expert. This means that either local SLR did not manage to align the IFOF bundles as closely as possible or that the pruning_thr was too small (which could be easily re-adjusted). Nonetheless, RecoBundles was able to detect a large amount of the IFOF. For the right IFOF, the model bundle had 698 streamlines, the RecoBundles bundle had 321, and the expert bundle had 546. For the left IFOF, the model bundle had 706 streamlines, the RecoBundles bundle 601 streamlines, and the RecoBundles bundle 847 bundles.

In the fifth and sixth rows, we see a denser recognition of the optic radiation again by RecoBundles. The right OR model bundle had 129 streamlines, RecoBundles had 413 streamlines, and expert bundle had 154 streamline. The left OR model bundle had 293 streamlines, RecoBundles bundle 413, and expert bundle 154 streamlines. 
Table 1

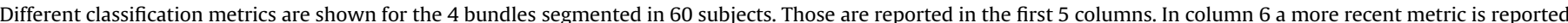

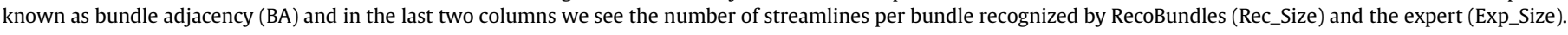

\begin{tabular}{|c|c|c|c|c|c|c|c|c|}
\hline & Sensitivity & Specificity & Accuracy & Precision & Jaccard & $\mathrm{BA}$ & Rec_Size & Exp_Size \\
\hline IFOF_L & $0.68 \pm 0.12$ & $1.0 \pm 0.0$ & $0.99 \pm 0.0$ & $0.26 \pm 0.08$ & $0.23 \pm 0.07$ & $0.53 \pm 0.09$ & $1010.26 \pm 176.34$ & $382.1 \pm 141.95$ \\
\hline IFOF_R & $0.69 \pm 0.11$ & $1.0 \pm 0.0$ & $0.99 \pm 0.0$ & $0.3 \pm 0.09$ & $0.26 \pm 0.07$ & $0.56 \pm 0.09$ & $1065.18 \pm 187.93$ & $449.05 \pm 155.63$ \\
\hline UNC_L & $0.85 \pm 0.13$ & $1.0 \pm 0.0$ & $1.0 \pm 0.0$ & $0.22 \pm 0.06$ & $0.21 \pm 0.06$ & $0.61 \pm 0.08$ & $492.8 \pm 81.65$ & $127.97 \pm 44.45$ \\
\hline UNC_R & $0.92 \pm 0.08$ & $1.0 \pm 0.0$ & $1.0 \pm 0.0$ & $0.27 \pm 0.07$ & $0.26 \pm 0.07$ & $0.68 \pm 0.07$ & $639.11 \pm 93.27$ & $186.92 \pm 62.16$ \\
\hline
\end{tabular}

Table 2

Sensitivity and Jaccard index calculated using spatial extent (SE). Notice the increase in comparison to the same metrics of Table 1 . Here we are counting the number of voxels which the streamlines pass. In the last two columns, we see the mean and standard deviation of total number of voxels traversed by the recognized bundles (SE_Rec_Size) and the bundles segmented by experts (SE_Exp_Size).

\begin{tabular}{lllll}
\hline & SE_Sensitivity & SE_Jaccard & SE_Rec_Size & SE_Exp_Size \\
\hline IFOF_L & $0.76 \pm 0.1$ & $0.31 \pm 0.08$ & $8627.18 \pm 1378.06$ & $3908.98 \pm 1274.15$ \\
IFOF_R & $0.77 \pm 0.09$ & $0.35 \pm 0.08$ & $8880.38 \pm 1430.95$ & $4490.02 \pm 1390.72$ \\
UNC_L & $0.88 \pm 0.11$ & $0.29 \pm 0.07$ & $3808.58 \pm 594.91$ & $1327.62 \pm 434.21$ \\
UNC_R & $0.94 \pm 0.07$ & $0.36 \pm 0.08$ & $4838.31 \pm 667.53$ & $1867.54 \pm 570.21$ \\
\hline
\end{tabular}

In the seventh row, we see a thicker model bundle generating a thinner than the expert's bundle but still the main and important corpus of the splenium (SPL) has been found (model 522, RecoBundles 195, and expert 642 streamlines).

In the last row, using the middle cerebellar peduncle (MCP) as the model bundle RecoBundles was able to detect a nearly equal amount of streamlines in comparison to the expert. However the frontal part of the MCP (around the pons) was not as dense as determined by the expert. However, this can be adjusted by changing the pruning_thr. The model bundle had 2378, RecoBundles generated 743 , and the expert generated 736 streamlines. Notice here that the model had 3 times more streamlines than the expert segmentation, but RecoBundles was not affected by that and it was able to extract MCP from the other participant.

Finally, these tractograms had about half a million streamlines each. We did not observe any reduction on the accuracy of the algorithm when we tried larger tractograms, for example, 1 million or 3 million streamlines. RecoBundles was always able to find the main corpus of the given bundle in efficient computation time.

\subsection{Adaptation to tumors/patient data}

In this section, we describe the application of RecoBundles in 11 tumor cases. The datasets were collected before brain surgery. The study was performed according to the guidelines of the Internal Review Board of the Center Hospitalier Universitaire de Sherbrooke (CHUS). We saw the first case in which RecoBundles detected a bundle going through a large tumor area mostly affecting the upper part of the CST in Fig. 1. Tracking is difficult in brains with large tumors because many of the bundles are either broken in shorter parts, or deformed, or non-existent. Even for an expert, it is difficult to segment such a bundle using manual virtual dissection. In this case (see Fig. 1), an expert neuroanatomist had to use extra information from an fMRI finger tapping experiment to make sure that the segmentation was accurate. Despite the difficulty of this dataset, RecoBundles was able to successfully recover the bundle as we showed in Garyfallidis et al. (2015a). The datasets used here are described in Appendix A.1 and Appendix A.2.

In Fig. 1, we showed a dataset with a tumor mostly affecting the

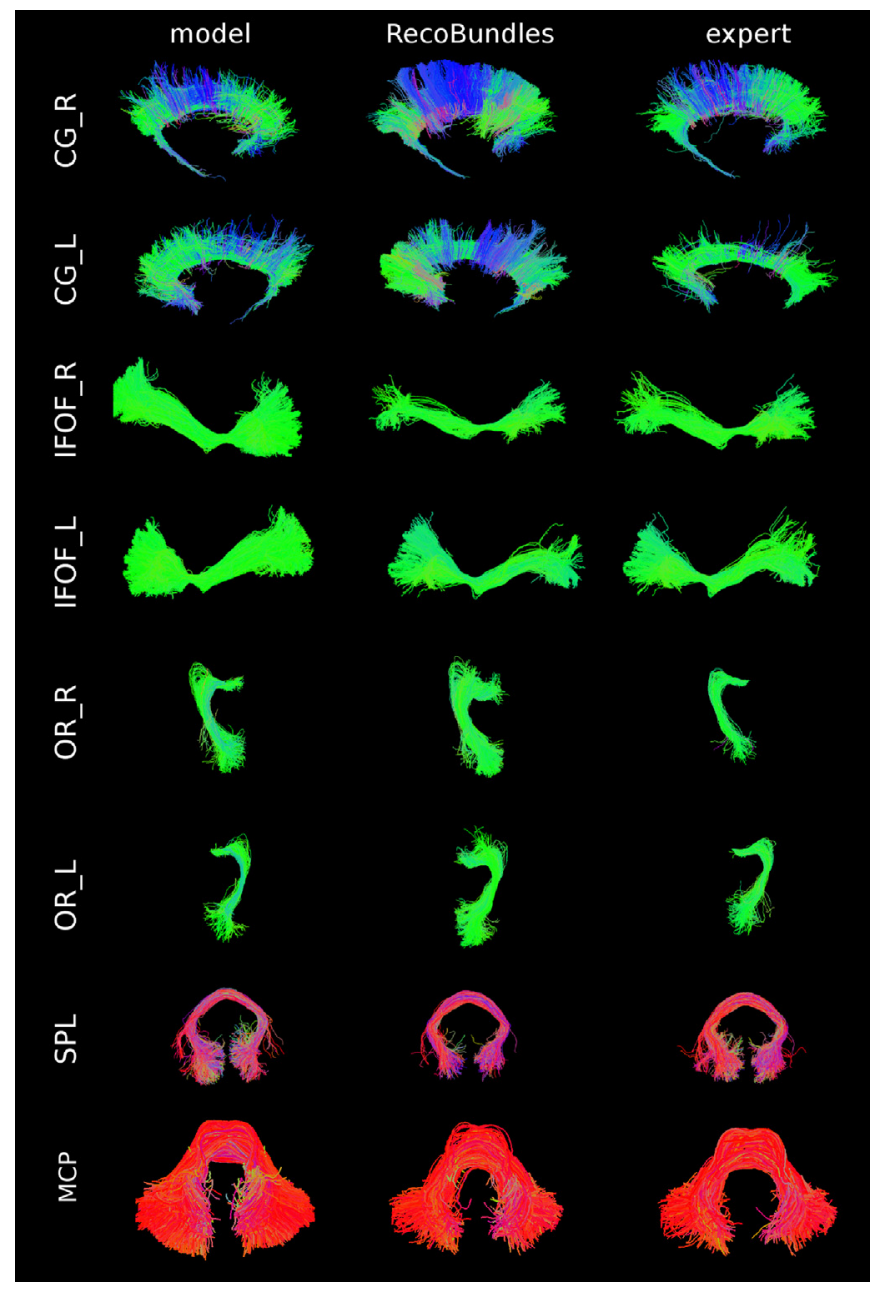

Fig. 6. Visual comparisons between RecoBundles' segmentation and expert's segmentation of different bundles. For RecoBundles we used the segmented bundles from a different participant than the target. The model bundles, $B_{M}^{i}$, are shown on the left column. The bundles segmented with RecoBundles using the model bundles as priors are shown in the middle $B_{A}^{i}$ and on the right we see the bundles segmented from experts $B_{E}^{i}$ using their knowledge of anatomy and a virtual dissection tool (here Trackvis). Notice how RecoBundles can recognize bundles equally well to the experts and is adaptive to different types of bundles as models. Notice also that the model is just a bundle from a different participant than the target and not generated from an atlas.

upper part of the CST. In this example shown in Fig. 7, a different dataset is provided and the tumor here is affecting the middle area of the CST. We show both affected and unaffected sides. The processing strategy used to generate this dataset is given in Appendix A.2.

Here, we used RecoBundles to extract two corticospinal tracts (CST) from a different brain with a large tumor (see Fig. 7). The 


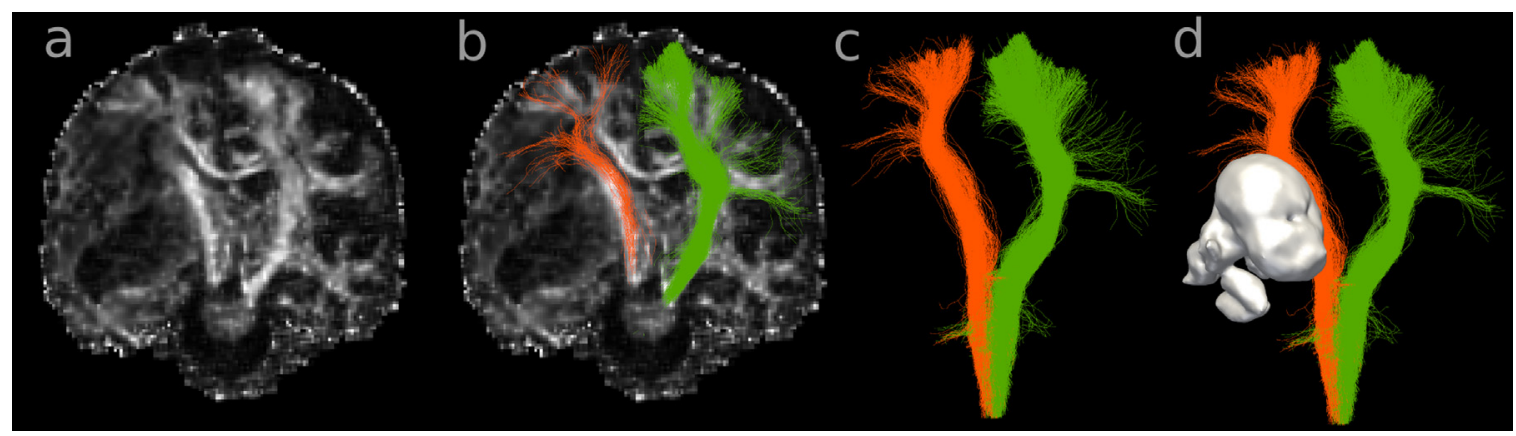

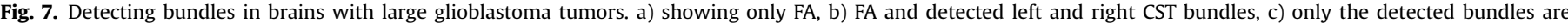
shown, and d) the bundles and a surface rendering of the large tumor is shown.

tumor can be seen using fractional anisotropy in Fig. 7a and it is also surface rendered in $7 \mathrm{~d}$. Tracking can be very difficult in tumor areas because of edema but also the tumor can add pressure and deform bundles. The deformation is shown also in Fig. 7b,c. In this case, we see in Fig. 7c,d that RecoBundles was successful in recognizing and extracting those bundles. The healthy CST bundles used as model bundles were generated from the HCP data and used during the ISMRM Tractography Challenge described in Appendix B. Two model bundles of healthy CST were used and the parameters for RecoBundles were reduction_thr $20 \mathrm{~mm}$, clust_thr $12 \mathrm{~mm}$, the SLR matrix size was $400 \times 400$, and pruning_thr $8 \mathrm{~mm}$.

Note that any image-based atlas technique requiring a parcellation and nonlinear registration will have a difficult time with such dataset with a large tumor. We confirmed this by trying to use connectivity-based tools e.g. TractQuerier (Wassermann et al., 2013) or Tracula (Yendiki et al., 2011) which were not capable of segmenting this dataset because the tumor was too large and the initial parcellation from Freesurfer was failing in the tumor's region. Nonetheless, RecoBundles was able to detect the bundle. This experiment illustrated the importance of using bundle models (in their streamline form) as shape priors to drive the recognition/ segmentation.

Next, we show 9 more cases showing the detection of affected and/or unaffected bundles (see Figs. 8 and 9). In Fig. 8, we show 3 new cases each from a different subject. For each case, one bundle of interest was chosen to highlight the local impact of the tumor on the brains connectivity. Bundles are displayed in green and red simply based on the hemisphere they are in, the bundles in the same hemisphere as the tumor are red, while the bundles in the opposite hemisphere of the tumor are green. The tumor is rendered in purple, and the FA is displayed in the background. The view was chosen to be in the most relevant axis for visualization purposes. In Fig. 8A, a 31 year old, right handed female presenting with epilepsy due to a right (or non-dominant) fronto-temporal insular anaplastic oligodendroglioma. In Fig. 8B, a 62 year old, right handed male presenting with cephalalgia due to a left (or dominant) temporal glioblastoma proximal to Wernickes area. In Fig. 8C, A 51 year old male presenting with epilepsy due to a right temporal grade 2 astrocytoma, originating from the right hippocampus.

In Fig. 8A (IFOF) and Fig. 8C (OR), the tumor is directly in the expected path of the bundle. In Fig. 8B (CST) the tumor is distant from the bundle of interest but has pushed and deformed the midupper part of the bundle (when compared to the other hemisphere). In all three labels, the general shape of the bundle remains the same, the displacement of the bundle is minor despite the size and proximity of the tumor.

Fig. 9 shows 6 new cases. Both these 6 cases and the previous cases were generated using deterministic tracking as described in
Appendix A.2. The same coloring scheme was used as in Fig. 8. Here we provide a short description of the 6 cases. In Fig. 9A, a 44 year old, right handed female presenting with epilepsy and speech deficits due to a left (or dominant) fronto-temporal glioblastoma. In Fig. 9B, a 35 year old, right handed male presenting with epilepsy due to a right (or nondominant) frontal glioblastoma. In Fig. 9C, a 41 year old, right handed male presenting with epilepsy due to a right (or nondominant) frontal glioblastoma. In Fig. 9D, A 29 year old, right handed female presenting with hemiparesis due to a left (or dominant) frontal grade 3 astrocytoma. The objective here was to determine if streamlines of the CG had been disrupted or displaced. In Fig. 9E, a 48 year old, right handed male presenting with epilepsy due to a right (or nondominant) temporal glioblastoma. The objective was to examine possible incoherent structure of the ILF. In Fig. 9F, a 64 year old, right handed female presenting with psychomotor retardation due to right or (nondominant) fronto-temporal glioblastoma. The primary objective here was to use RecoBundles to visualize any distortions to the SLF, which were anticipated by the neurocognitive deficits.

In Fig. 9, each column is showing the same bundle to demonstrate how tumors can affect the same pathway in different cases. Also for each column the same model bundle is used. In the first column, the SLF is pushed in 2 different directions, either dorsally as shown in Fig. 9A or caudally shown in Fig. 9B. It is important to mention that the latter image was flipped to facilitate visualization, originally the tumor is on the right side. In Fig. 9C the CG being either disrupted at mid-length and in Fig. 9D pushed medially. The last column shows the ILF. In Fig. 9E the tumor seems to have a minor impact on the bundle (with a slight lateral displacement). In Fig. 9F, the integrity of the bundle is highly compromised, its volume and path are significantly affected.

The CST, OR and SLF were recognized using model bundles from the ISMRM 2015 white matter challenge. The rest of the bundles, i.e. CG, IFOF and ILF bundles were recognized using the corresponding model bundles from subject 100,307 of the HCP project as these bundles were not available in ISMRM 2015 challenge. The data description for the challenge is available in Appendix B and for subject 100,307 in Appendix D. The parameters used for RecoBundles in Figs. 8 and 9 were clust_thr $16 \mathrm{~mm}$, matrix_size $400 \times 400$ and transformation is rigid. The pruning_thr are slightly different. For OR and IFOF is $7 \mathrm{~mm}$. For CG, ILF and CST is $6 \mathrm{~mm}$ and for SLF the pruning_thr is $5 \mathrm{~mm}$.

In this section, we illustrated the adaptability of RecoBundles in segmenting bundles despite variation in shape, length, volume and distance between bundles and tumors in 11 cases of patients with brain tumors (see Figs. 1, 7-9). 


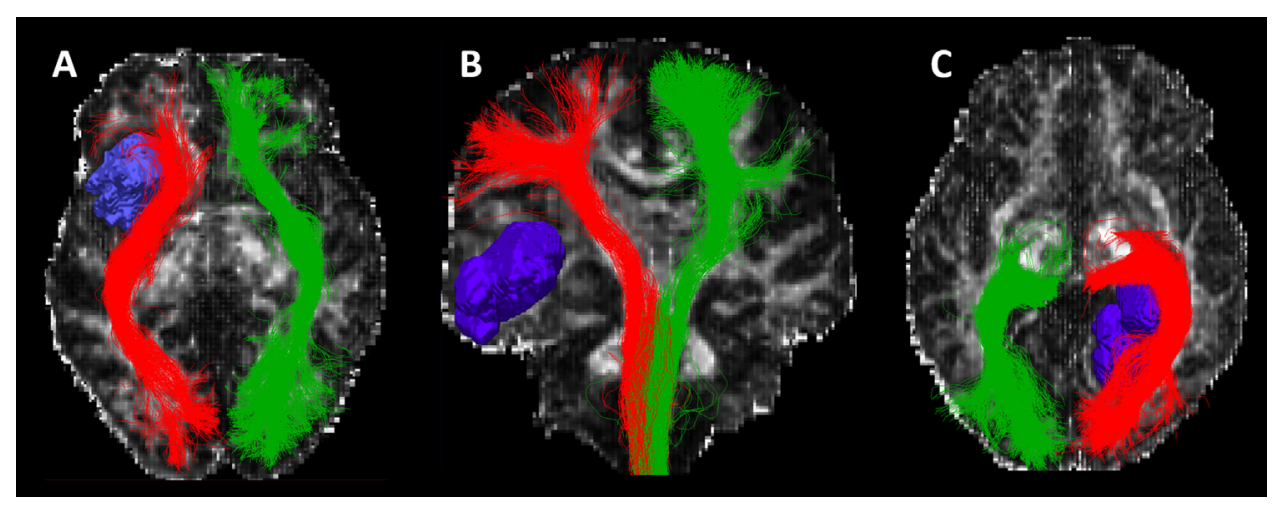

Fig. 8. Detecting bundles in brains with large glioblastoma tumors in 3 cases (A-C). FA is displayed in the background of all cases. The tumors are depicted with purple. In A and $C$, the tumor is directly affecting the path of bundles IFOF and OR respectively. In B, the tumor is deforming the CST bundle from distance without directly infiltrating the bundle.

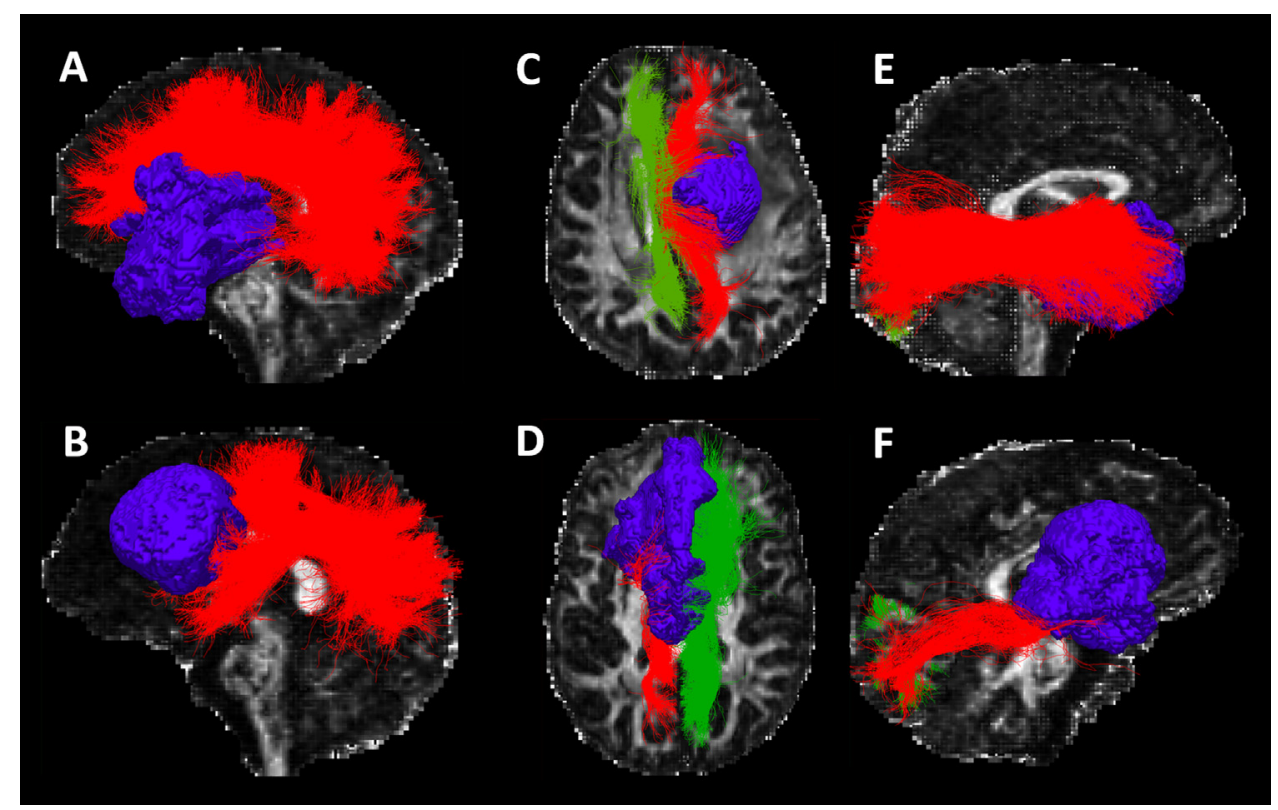

Fig. 9. RecoBundles detecting bundles in brains with large glioblastoma tumors in 6 cases (A-F). Two different tumors affecting the SLF are shown in A and B. Two different tumors affecting the CG are shown in C and D. Finally, two different tumors affecting the ILF are shown in E and F. Pictures taken from most relevant axis that can facilitate visualization of bundles and tumors.

\section{Discussion}

\subsection{Overview}

We showed in this manuscript how RecoBundles was able to recognize bundles of different shapes, length and type both in healthy and patient tractograms. We also measured its accuracy quantitatively with simulations of deformations applied in healthy bundles and we showed qualitative results of bundles deformed by tumors. RecoBundles was able to detect deformed and often interrupted bundles going through or around tumor areas (see Figs. 1, 7-9). The quantitative and qualitative experiments showed that RecoBundles is adaptive to shape changes, incomplete datasets and different quality of tractography output. For the uncinate and inferior fronto-occipital fasciculi we were able to measure the sensitivity of the algorithm across 60 subjects to be on average between 76 and $94 \%$, providing similar results to the expert segmentations (see Fig. 5).
Notice that in order to generate the results shown in this paper, we did not have to use any classification techniques or advanced machine learning techniques e.g. deep learning (LeCun et al., 2015) to perform the segmentations. All "the learning" was generated a) by sparsifying the tractograms using QuickBundles and b) using a straightforward local minimization with streamline-based linear registration (SLR), to directly match the model bundles to the target bundles. Now that we have a robust and efficient technique, we can use it as a first step to generate fast segmentations in thousands of datasets. One could then train a deep learning or similarly advanced algorithm that requires large training datasets of bundles which are currently not available.

Recent techniques to perform bundle segmentation (Wassermann et al., 2013; Tunç et al., 2014, 2016; O'Donnell et al., 2017; Wu et al., 2012; Guevara et al., 2011) have appeared in similar spirit to RecoBundles. They all differ methodologically but are developed towards the same goal, trying to extract known white matter bundles using a set of prior models. It is too early to 
pinpoint advantages/disadvantages of these techniques but they should be systemically compared to asses quality, speed and robustness to different input tractograms.

In this paper, we used the MDF distance for all the different computations between streamlines i.e. clustering, registration and pruning. However, RecoBundles can be easily modified to use other distances proposed in the literature such as those used in O'Donnell et al. (2017); Yoo et al. (2015); Labra et al. (2016) and Gori et al. (2016).

The difference between QuickBundles and RecoBundles is that while QuickBundles is a fast unsupervised method for simplifying massive tractograms, RecoBundles is a fast supervised method that requires a bundle model as input and detects relevant bundles in a given tractogram. RecoBundles is a method that is easy to set up and can be adjusted according to the neuroanatomists' needs by adjusting the pruning threshold. Allowing for this free parameter allows to the neuroanatomist to examine the surrounding region of a bundle (see Fig. 3). Therefore, RecoBundles can be easily integrated in new virtual dissection tools (Porro-Muñoz et al., 2015) and facilitate the segmentation process.

It currently takes a couple of minutes to have more than 20 different bundles segmented in tractograms of one million streamlines. The part that takes most of the time is the initial clustering in Phase A (see Fig. 2) which we are currently working to shorten; see preliminary results here Garyfallidis et al. (2016).

RecoBundles will be able to i) help create large collections of streamline-based bundle atlases which are currently not available because of the large amount of work needed to manually segment many bundles in multiple subjects and ii) help in tractometry studies. In tractometry (Bells et al., 2011; Yeatman et al., 2012; Dayan et al., 2016) the statistical analysis takes place in specific bundles and an automatic segmentation tool such as RecoBundles that allow to extract bundles directly in native space can help make tractometry more efficient and more automated.

RecoBundles can potentially use model bundles that are generative. So, one could potentially simulate or even draw a hypothetical bundle in a computer aided design (CAD) program and detect this bundle using RecoBundles in an existing tractogram. Moreover, one can include broken or shorter parts in a model bundle to allow a more elaborate dissection. This for example would be beneficial for patient data where bundles are potentially completely cut, along their length, in one or more parts. As future work we would like to recognize the superficial short association bundles found in Guevara et al. (2017) and see which of them can be found in lower quality datasets.

\subsection{Disabling SLR}

In this work, we studied the capablies of a method that is $100 \%$ streamline-based and $100 \%$ linear. However, if one wants to boost the segmentation precision even more, then one could disable local streamline-based linear registration (SLR), register the images using standard nonlinear registration techniques, as those found in ANTs (Avants et al., 2009) and DIPY (Garyfallidis et al., 2014), and then apply the deformation fields to the streamlines and the deformed streamlines as input to RecoBundles. This technique will give higher classification accuracy in healthy subjects since the matching is already heavily resolved. Then it is only a matter of clustering and pruning. However, if one is interested in studying bundles of patients with severe brain damage, for example TBI patients, large concussions or tumors where nonlinear registration is difficult or fails then we suggest to use the SLR which is our default choice in this paper.

In Maier-Hein et al. (2016), a minimalistic version of RecoBundles was used for the evaluation of the ISMRM 2015 challenge. This version of RecoBundles with SLR (global and local) disabled was used because the tractograms were from the same participant. However, the tractograms had deformations in comparison to the ground truth. So, only clustering and pruning were used. Even in its minimal version, were registration is disabled, RecoBundles can be used to solve important assignment problems such as finding which generated bundles identify with the ground truth. RecoBundles was used to score teams as tractograms were not perfectly aligned with the ground truth as ROI and connectivity based methods were not precise enough to find the correct streamlines. RecoBundles was generating much denser and closer to the ground truth bundles than standard ROI-based (connectivity) extraction. For more details see Maier-Hein et al. (2016).

\subsection{Beyond the human brain}

RecoBundles has no prior knowledge about if the input tractogram is a human brain tractogram or any other brain. There is no dependence to a specific atlas. Therefore, although we have not performed any experiments yet, in theory the same technique can be used to segment bundles in tractograms of other animal brains as long as expert anatomists have manually segmented some bundles from the same species that can be used as models. In the future, we would like to examine that possibility and use RecoBundles to create bundle atlases (Garyfallidis et al., 2015b) from the tractograms of other mammalian brains.

\section{Conclusion}

RecoBundles is a new powerful and efficient method for recognizing, mining, searching and investigating bundles in tractograms of the same or different subjects or tractography algorithms. The input and outputs of RecoBundles are streamlines and all the processing takes place in the space of streamlines. Hence, it can easily be integrated in any neuroimaging pipeline.

This gives great hope to use streamline-based algorithms and bundle analytics for further research in neurological disorders. For example, we will assess bundles in Alzheimer's patients with white matter microlesions, gray matter atrophy and enlarged ventricles, and measure shape changes in homologous bundles during tractometry studies. RecoBundles will be publicly available in DIPY. ${ }^{2}$

\section{Acknowledgments}

We are grateful to the Établissement Nouveau Chercheur FQNRT program, the University-Industry NSERC CREATE Medical Image Analysis program, the Samuel de Champlain France-Québec program, a University Research Chair (SCC) and Prof. Descoteaux's Research Chair in Neuroinformatics for funding this research. We also thank Prof. David Fortin and Prof. Kevin Whittingstall for sharing some of the USherbrooke imaging data. Finally, we are grateful to the Indiana University for providing Prof. Garyfallidis' startup fund.

\section{Appendix A. USherbrooke data}

All subjects were scanned according to the guidelines of the Internal Review Board of the Center Hospitalier Universitaire de Sherbrooke (CHUS). Imaging data were acquired using a $1.5 \mathrm{~T}$

\footnotetext{
2 http://dipy.org.
} 
SIEMENS Magnetom (Vision). For both the datasets described below, in Sections Appendix A.2 and Appendix A.1, the same protocol was used for the diffusion MRI and high resolution anatomical T1-weighted images. Head motion was minimized using cushions. Each session started by a dMRI acquisition and then with an anatomical T1-weighted $1 \mathrm{~mm}$ isotropic MPRAGE (TR/TE 6.57/2.52 ms) acquisition. Diffusion images were acquired using the single-shot echo-planar imaging (EPI) multi-direction diffusion-weighted sequence. To reduce susceptibility distortions, GRAPPA parallel imaging was employed with an acceleration factor of 2. A 6/8 partial Fourier factor was used and 64 diffusion-weighted images were acquired along uniformly distributed directions using a b-value of $1000 \mathrm{~s} / \mathrm{mm}^{2}$. A single $\mathrm{b}=0 \mathrm{~s} /$ $\mathrm{mm}^{2}$ S0 image was also acquired. Other imaging parameters were matrix size of $128 \times 128,2 \mathrm{~mm}$ isotropic spatial resolution, TR/TE $11,000 / 98 \mathrm{~ms}$, resulting in a $12 \mathrm{~min}$ acquisition.

\section{Appendix A.1. USherbrooke 1.5T data}

Diffusion data were upsampled to $1 \mathrm{~mm}$ isotropic resolution using a trilinear interpolation (Tournier et al., 2012; Smith et al., 2012; Girard et al., 2014). The T1-weighted image was registered to a $1 \mathrm{~mm}$ isotropic DWI using ANTs (Avants et al., 2008). Quality control was done to make sure the registration was done robustly by manual inspection. The Fractional Anisotropy (FA) map and color-FA were overlaid on the T1-weighted image to make sure optimal alignment between images. Diffusion tensor estimation and corresponding FA were estimated using DIPY (Garyfallidis et al., 2014). From this, the single fiber response function was estimated from all FA values above a threshold of 0.7. This single fiber response was used as input to spherical deconvolution (Tournier et al., 2007) to compute the fiber orientation distribution function (fODF) (Descoteaux et al., 2009) at every voxel of the brain with a maximal spherical harmonics order of 8 and the default parameters. We used streamline deterministic tractography, the streamtrack MRtrix command (Tournier et al., 2012) on the field of FODs using multiple seeding and default tracking parameters (step size $0.2 \mathrm{~mm}$, minimum/maximum streamline length 10 / $200 \mathrm{~mm}$, minimum radius of curvature $1 \mathrm{~mm}$, fODF amplitude cutoff at 0.1 ) to reconstruct whole brain tractography datasets with 500,000 streamlines.

\section{Appendix A.2. Tumor data}

We used FOD reconstruction of order 8 as in the previous section and a deterministic tracking with step size 0.5 , and FA value of 0.12 as stopping criterion to generate around 1 million streamlines using MRtrix command (Tournier et al., 2012). We seeded to generate 1 million streamlines but after removing short streamlines of length less than $20 \mathrm{~mm}$, only $450-550$ thousand streamlines survived.

\section{Appendix B. ISMRM Challenge data}

The set of ground truth fiber bundles was designed to cover the whole brain and feature many of the relevant configurations such as crossing, kissing, twisting and fanning fibers, thus representing the morphology of real in vivo fiber bundles. The process to obtain these bundles consists of three steps. First, a whole brain global tractography is performed on a high quality diffusion-weighted image. Then, 25 major bundles are manually extracted from the resulting tractogram. In the third step, these bundles are then refined to obtain smooth and well defined bundles. The full and detailed process is described in Maier-Hein et al. (2016).

\section{Appendix C. BIL\&GIN diffusion data}

Diffusion-weighted images were previously acquired for 60 healthy right-handed participants (30 female, mean age = 30.1, age range $=20-53$ ) belonging to the BIL\&GIN database (Brain Imaging of Lateralization by the Groupe d'Imagerie Neurofonctionnelle; Mazoyer et al. (2016)). All the subjects gave written informed consent to participate in the study, which was approved by the local ethics committee (CCPRB Basse- Normandie). Imaging was performed on a Philips Achieva 3 T MRI scanner using a single-shot spin-echo echoplanar sequence with 21 non-collinear diffusion gradient directions $\left(\mathrm{b}=1000 \mathrm{~s} / \mathrm{mm}^{2}\right)$. Seventy axial slices parallel to the AC-PC plane were acquired from the bottom of the cerebellum to the top of the brain. The following imaging parameters were used: TR $=8500$ ms, $\mathrm{TE}=81 \mathrm{~ms}$, angle $=90$, SENSE reduction factor $=2.5$, FOV $224 \mathrm{~mm}$, acquisition matrix $112 \times 112,2 \mathrm{~mm}^{3}$ isotropic voxel. The series of 21 directions was acquired twice by reversing the gradients polarity, for a total of 42 diffusion-weighted volumes. To improve the signal-tonoise ratio, a second series of $42 \mathrm{vol}$ was acquired leading to a total acquisition time of $15 \mathrm{~min} 30 \mathrm{~s}$.

The raw diffusion images were corrected for eddy current distortion using FSL (Smith et al., 2004) and processed with the Diffusion Toolkit software package to obtain the local tensor orientation estimates and fractional anisotropy maps and perform fiber tracking. Deterministic whole-brain fiber tracking was performed in the native space of each individual using the Fiber Assignment by Continuous Tracking algorithm (Mori et al., 1999) with stopping criteria of 0.2 fractional anisotropy and a 45 angular threshold. Tracking was initiated by seeding from all voxels in the volume to generate the streamlines. This produced a 3D reconstruction of streamlines in the whole brain, namely a tractogram, which can then be segmented into anatomically defined bundles. For the complete description of the manual virtual dissection strategy see Hau et al. (2016).

\section{Appendix D. HCP processing and dissection strategy}

Subjects with IDs 100,307, 210,011, and 152,831 from the Human Connectome Project (HCP) dataset (Van Essen et al., 2013) were used. This dMRI acquisition (Sotiropoulos et al., 2013) has $1.25 \mathrm{~mm}$ isotropic resolution, with $3 \mathrm{~b}$-values $\left(1000,2000,3000 \mathrm{~s} / \mathrm{mm}^{2}\right)$ and a total of 270 gradient directions (90 per shell) and $18 \mathrm{~b}=0$ images. Fiber ODFs of order 8 were reconstructed with DIPY and probabilistic partial volume estimation maps with FSL fast (Zhang et al., 2001) to extract the white-matter/gray-matter (wm/gm) interface and include/ exclusion probabilistic regions to run the anatomically-constrained particle filter probabilistic tractography algorithm of Girard et al. 
(2014) with 10 seeds per voxel of the wm/gm interface and other default parameters, as recommended in the paper. Subject 100,307 was used in section 3.3 and the other two subjects were used in section 3.2.2.

Neuroanatomical expert dissection strategy. Cingulum left and right. A single sagittal ROI of the cingulum capturing the anterior to posterior connectivity was utilized, two planar not region were utilized in the midline to exclude commissural connectivity, as well as laterally to exclude thalamic projections, as reflected in Rojkova et al. (2016).

Inferior fronto-occipital fasciculus (IFOF) left and right. A two ROI approach was taken for each IFOF. A hand-drawn coronal ROI capturing the projections leaving from the floor of the external capsule was placed in the frontal lobe and utilized in conjunction with a hand-drawn coronal ROI within the occipital lobe to capture connectivity coming from the inferior and medial surfaces.

Optic radiation (OR) left and right. A $2 \mathrm{~mm}$ spherical was coronally placed in the posterior portion of the thalamus, lateral and superior to the fimbria of the hippocampus. This was utilized in conjunction with the an occipital lobe ROI to obtain the optic radiation projections from both hemispheres.

Splenium of the corpus callosum (SPL). Two ROIs were placed coronally at the level of the splenium of the corpus callosum in the occipital lobe in somewhat analogous positions to capture the homotopic commissural connectivity of the forceps major or splenium.

The middle cerebellar peduncle (MCP) was filtered from the tractograms using ROIs near the pons capturing the left and right sides of the MCP. All bundles were dissected using Trackvis and well established virtual dissection strategies as mentioned in Catani et al. (2002b).

\section{References}

Avants, B.B., Epstein, C.L., Grossman, M., Gee, J.C., 2008. Symmetric diffeomorphic image registration with cross-correlation: evaluating automated labeling of elderly and neurodegenerative brain. Med. image Anal. 12 (1), 26-41.

Avants, B.B., Tustison, N., Song, G., 2009. Advanced normalization tools (ants). Insight J. 2, 1-35.

Avants, B.B., Tustison, N.J., Song, G., Cook, P.A., Klein, A., Gee, J.C., 2011. A reproducible evaluation of ants similarity metric performance in brain image registration. Neuroimage 54 (3), 2033-2044.

Bells, S., Cercignani, M., Deoni, S., Assaf, Y., Pasternak, O., Evans, C., Leemans, A., Jones, D., 2011. Tractometry-comprehensive multi-modal quantitative assessment of white matter along specific tracts. Proc. ISMRM vol. 678; 2011.

Catani, M., Howard, R.J., Pajevic, S., Jones, D.K., 2002a. Virtual in vivo interactive dissection of white matter fasciculi in the human brain. Neuroimage 17 (1), 77-94.

Catani, M., Howard, R.J., Pajevic, S., Jones, D.K., 2002b. Virtual in vivo interactive dissection of white matter fasciculi in the human brain. Neurolmage 17, 77-94.

Chamberland, M., Whittingstall, K., Fortin, D., Mathieu, D., Descoteaux, M., 2014. Real-time multi-peak tractography for instantaneous connectivity display. Front. Neuroinf 8, 59.

Chamberland, M., Scherrer, B., Prabhu, S.P., Madsen, J., Fortin, D., Whittingstall, K., Descoteaux, M., Warfield, S.K., Jan 2017. Active delineation of Meyer's loop using oriented priors through MAGNEtic tractography (MAGNET). Hum. Brain Mapp. 38 (1), 509-527.

Côté, M.-A., Girard, G., Boré, A., Garyfallidis, E., Houde, J.-C., Descoteaux, M., 2013. Tractometer: towards validation of tractography pipelines. Med. image Anal. 17 (7), 844-857.

Dayan, M., Monohan, E., Pandya, S., Kuceyeski, A., Nguyen, T.D., Raj, A., Gauthier, S. A., 2016. Profilometry: a new statistical framework for the characterization of white matter pathways, with application to multiple sclerosis. Hum. Brain Mapp. 37 (3), 989-1004.

Descoteaux, M., Deriche, R., Knösche, T.R., Anwander, A., Febuary 2009. Deterministic and probabilistic tractography based on complex fibre orientation distributions. IEEE Trans. Med. Imaging 28 (2), 269-286.

Durrleman, S., Fillard, P., Pennec, X., Trouvé, A., Ayache, N., 2011. Registration, atlas estimation and variability analysis of white matter fiber bundles modeled as currents. NeuroImage 55 (3), 1073-1090.

Garyfallidis, E., Brett, M., Correia, M.M., Williams, G.B., Nimmo-Smith, I., 2012. QuickBundles, a method for tractography simplification. Front. Neurosci. 6 (175), 1-13.

Garyfallidis, E., Brett, M., Amirbekian, B., Rokem, A., Van Der Walt, S., Descoteaux, M., Nimmo-Smith, I., 2014. Dipy, a library for the analysis of diffusion mri data. Front. Neuroinformatics 8 (8).

Garyfallidis, E., Ct, M.-A., Hau, J., Perchey, G., Petit, L., Cunnanne, S.C., Descoteaux, M., 2015a. Recognition of bundles in healthy and severely diseased brains, International Society for Magnetic Resonance Imaging (ISMRM).

Garyfallidis, E., Ocegueda, O., Wassermann, D., Descoteaux, M., 2015b. Robust and efficient linear registration of white-matter fascicles in the space of streamlines. NeuroImage 117, 124-140.

Garyfallidis, E., Ct, M.-A., Rheault, F., Descoteaux, M., 2016. Quickbundlesx: sequential clustering of millions of streamlines in multiple levels of detail at record execution time, International Society for Magnetic Resonance Imaging (ISMRM).

Girard, G., Whittingstall, K., Deriche, R., Descoteaux, M., May 2014. Towards quantitative connectivity analysis: reducing tractography biases. NeuroImage 98 (1), $266-278$.
Gori, P., Colliot, O., Marrakchi-Kacem, L., Worbe, Y., Fallani, F.D.V., Chavez, M., Poupon, C., Hartmann, A., Ayache, N., Durrleman, S., 2016. Parsimonious approximation of streamline trajectories in white matter fiber bundles. IEEE Trans. Med. Imaging 99.

Guevara, P., Duclap, D., Poupon, C., Marrakchi-Kacem, L., Houenou, J., Leboyer, M., Mangin, J.-F., 2011. Segmentation of short association bundles in massive tractography datasets using a multi-subject bundle atlas, Progress in Pattern Recognition, Image Analysis, Computer Vision, and Applications. Springer, pp. 701-708.

Guevara, P., Duclap, D., Poupon, C., Marrakchi-Kacem, L., Fillard, P., Le Bihan, D., Leboyer, M., Houenou, J., Mangin, J.-F., 2012. Automatic fiber bundle segmentation in massive tractography datasets using a multi-subject bundle atlas. Neuroimage 61 (4), 1083-1099.

Guevara, M., Román, C., Houenou, J., Duclap, D., Poupon, C., Mangin, J.F., Guevara, P. 2017. Reproducibility of superficial white matter tracts using diffusion-weighted imaging tractography. NeuroImage 147, 703-725.

Hagmann, P., Cammoun, L., Gigandet, X., Meuli, R., Honey, C.J., Wedeen, V.J., Sporns, O., Jul. 2008. Mapping the structural core of human cerebral cortex. PLoS Biol. 6 (7), e159.

Hau, J., Sarubbo, S., Perchey, G., Crivello, F., Zago, L., Mellet, E., Jobard, G., Joliot, M. Mazoyer, B.M., Tzourio-Mazoyer, N., et al., 2016. Cortical terminations of the inferior fronto-occipital and uncinate fasciculi: anatomical stem-based virtual dissection. Front. Neuroanat. 10.

Jin, Y., Shi, Y., Zhan, L., Gutman, B.A., de Zubicaray, G.I., McMahon, K.L., Wright, M.J. Toga, A.W., Thompson, P.M., 2014. Automatic clustering of white matter fibers in brain diffusion mri with an application to genetics. Neuroimage 100, 75-90.

Jones, D.K., Knösche, T.R., Turner, R., 2013. White matter integrity, fiber count, and other fallacies: the do's and don'ts of diffusion mri. Neuroimage 73, 239-254.

Labra, N., Figueroa, M., Guevara, P., Duclap, D., Hoeunou, J., Poupon, C., Mangin, J.-F., 2013. Gpu-based acceleration of an automatic white matter segmentation algorithm using cuda, Engineering in Medicine and Biology Society (EMBC), 2013 35th Annual International Conference of the IEEE. IEEE, pp. 89-92.

Labra, N., Guevara, P., Duclap, D., Houenou, J., Poupon, C., Mangin, J.-F., Figueroa, M. 2016. Fast automatic segmentation of white matter streamlines based on a multi-subject bundle atlas. Neuroinformatics, 1-16.

LeCun, Y., Bengio, Y., Hinton, G., 2015. Deep learning. Nature 521 (7553), 436-444.

Maier-Hein, K., Neher, P., Houde, J.-C., Cote, M.-A., Garyfallidis, E., Zhong, J., Chamberland, M., Yeh, F.-C., Lin, Y.C., Ji, Q., Reddick, W.E., Glass, J.O., Chen, D.Q., Feng, Y., Gao, C., Wu, Y., Ma, J., Renjie, H., Li, Q., Westin, C.-F., DeslauriersGauthier, S., Gonzalez, J.O.O., Paquette, M., St-Jean, S., Girard, G., Rheault, F., Sidhu, J., Tax, C.M.W., Guo, F., Mesri, H.Y., David, S., Froeling, M., Heemskerk, A M., Leemans, A., Bore, A., Pinsard, B., Bedetti, C., Desrosiers, M., Brambati, S., Doyon, J., Sarica, A., Vasta, R., Cerasa, A., Quattrone, A., Yeatman, J., Khan, A.R., Hodges, W., Alexander, S., Romascano, D., Barakovic, M., Auria, A., Esteban, O. Lemkaddem, A., Thiran, J.-P., Cetingul, H.E., Odry, B.L., Mailhe, B., Nadar, M., Pizzagalli, F., Prasad, G., Villalon-Reina, J., Galvis, J., Thompson, P., Requejo, F., Laguna, P., Lacerda, L., Barrett, R., Dell'Acqua, F., Catani, M., Petit, L., Caruyer, E. Daducci, A., Dyrby, T., Holland-Letz, T., Hilgetag, C., Stieltjes, B., Descoteaux, M. 2016. Tractography-based Connectomes Are Dominated by False-positive Connections, bioRxiv.

Mazoyer, B., Mellet, E., Perchey, G., Zago, L., Crivello, F., Jobard, G., Delcroix, N., Vigneau, M., Leroux, G., Petit, L., et al., 2016. Bil\&gin: a neuroimaging, cognitive, behavioral, and genetic database for the study of human brain lateralization. NeuroImage 124, 1225-1231.

Mori, S., Crain, B.J., Chacko, V.P., Van Zijl, P.C.M., Feb. 1999. Three-dimensional tracking of axonal projections in the brain by magnetic resonance imaging. Ann. Neurol. 45 (2), 265-269.

Olivetti, E., Sharmin, N., Avesani, P., 2016. Alignment of tractograms as graph 
matching. Front. Neurosci. 10, 554.

Özarslan, E., Koay, C.G., Shepherd, T.M., Komlosh, M.E., İrfanoğlu, M.O., Pierpaoli, C., Basser, P.J., 2013. Mean apparent propagator (map) mri: a novel diffusion imaging method for mapping tissue microstructure. Neurolmage 78, 16-32.

O'Donnell, L.J., Westin, C.-F., 2007. Automatic tractography segmentation using a high-dimensional white matter atlas. Med. Imaging, IEEE Trans. 26 (11) 1562-1575.

O'Donnell, L.J., Westin, C.-F., Golby, A.J., 2009. Tract-based morphometry for white matter group analysis. NeuroImage 45 (3), 832-844.

O'Donnell, L.J., Suter, Y., Rigolo, L., Kahali, P., Zhang, F., Norton, I., Albi, A., Olubiyi, O., Meola, A., Essayed, W.I., et al., 2017. Automated white matter fiber tract identification in patients with brain tumors. NeuroImage Clin. 13, 138-153.

Porro-Muñoz, D., Olivetti, E., Sharmin, N., Nguyen, T.B., Garyfallidis, E., Avesani, P. 2015. Tractome: a visual data mining tool for brain connectivity analysis. Data Min. Knowl. Discov., 1-22.

Renauld, E., Descoteaux, M., Bernier, M., Garyfallidis, E., Whittingstall, K., 2016. Semi-automatic segmentation of optic radiations and lgn, and their relationship to eeg alpha waves. PLoS One 11 (7), e0156436.

Rojkova, K., Volle, E., Urbanski, M., Humbert, F., DellAcqua, F., de Schotten, M.T., 2016. Atlasing the frontal lobe connections and their variability due to age and education: a spherical deconvolution tractography study. Brain Struct. Funct. 221 (3), 1751-1766.

Siless, V., Chang, K., Fischl, B., Yendiki, A., 2016. Hierarchical clustering of tractography streamlines based on anatomical similarity, International Conference on Medical Image Computing and Computer-assisted Intervention. Springer, pp. 184-191.

Smith, S.M., Jenkinson, M., Woolrich, M.W., Beckmann, C.F., Behrens, T.E., JohansenBerg, H., Bannister, P.R., De Luca, M., Drobnjak, I., Flitney, D.E., et al., 2004. Advances in functional and structural $\mathrm{mr}$ image analysis and implementation as fsl. Neuroimage 23, S208-S219.

Smith, R.E., Tournier, J.-D., Calamante, F., Connelly, A., Jun. 2012. Anatomicallyconstrained tractography: improved diffusion MRI streamlines tractography through effective use of anatomical information. NeuroImage 62 (3), 1924-1938.

Sotiropoulos, S.N., Jbabdi, S., Xu, J., Andersson, J.L., Moeller, S., Auerbach, E.J., Glasser, M.F., Hernandez, M., Sapiro, G., Jenkinson, M., et al., 2013. Advances in diffusion mri acquisition and processing in the human connectome project. Neuroimage 80, 125-143.

Thomas, C., Frank, Q.Y., Irfanoglu, M.O., Modi, P., Saleem, K.S., Leopold, D.A., Pierpaoli, C., 2014. Anatomical accuracy of brain connections derived from diffusion mri tractography is inherently limited. Proc. Natl. Acad. Sci. 111 (46), 16574-16579.

Tournier, J.-D., Calamante, F., Connelly, A., 2007. Robust determination of the fibre orientation distribution in diffusion mri: non-negativity constrained super-resolved spherical deconvolution. NeuroImage 35 (4), 1459-1472.
Tournier, J.-D., Calamante, F., Connelly, A., Mar. 2012. MRtrix: diffusion tractography in crossing fiber regions. Int. J. Imaging Syst. Technol. 22 (1), 53-66.

Tunç, B., Parker, W.A., Ingalhalikar, M., Verma, R., 2014. Automated tract extraction via atlas based adaptive clustering. Neuroimage 102, 596-607.

Tunç, B., Ingalhalikar, M., Parker, D., Lecoeur, J., Singh, N., Wolf, R.L., Macyszyn, L., Brem, S., Verma, R., 2016. Individualized map of white matter pathways: connectivity-based paradigm for neurosurgical planning. Neurosurgery 79 (4), 568-577.

Van Essen, D.C., Smith, S.M., Barch, D.M., Behrens, T.E.J., Yacoub, E., Ugurbil, K., Oct. 2013. The WU-Minn human connectome project: an overview. NeuroImage 80, 62-79.

Wang, R., Benner, T., Sorensen, A., Wedeen, V., 2007a. Diffusion toolkit: a software package for diffusion imaging data processing and tractography. Proceedings of the International Society for Magnetic Resonance in Medicine vol. 15; 2007, p. 3720.

Wang, R., Benner, T., Sorensen, A., Wedeen, V., 2007b. Diffusion toolkit: a software package for diffusion imaging data processing and tractography. Proc Intl Soc Mag Reson Med vol. 15; 2007b.

Wassermann, D., Makris, N., Rathi, Y., Shenton, M., Kikinis, R., Kubicki, M., Westin, C.-F., 2013. On describing human white matter anatomy: the white matter query language, Medical Image Computing and Computer-assisted Intervention-MICCAI 2013. Springer, pp. 647-654.

Wassermann, D., Makris, N., Rathi, Y., Shenton, M., Kikinis, R., Kubicki, M., Westin, C.-F., 2016. The white matter query language: a novel approach for describing human white matter anatomy. Brain Struct. Funct., 1-17.

Wu, X., Xie, M., Zhou, J., Anderson, A.W., Gore, J.C., Ding, Z., 2012. Globally optimized fiber tracking and hierarchical clustering a unified framework. Magn. Reson. Imaging 30 (4), 485-495.

Yeatman, J.D., Dougherty, R.F., Myall, N.J., Wandell, B.A., Feldman, H.M., 112012. Tract profiles of white matter properties: automating fiber-tract quantification. PLoS One 7 (11), e49790.

Yendiki, A., Panneck, P., Srinivasan, P., Stevens, A., Zöllei, L., Augustinack, J., Wang, R., Salat, D., Ehrlich, S., Behrens, T., et al., 2011. Automated probabilistic reconstruction of white-matter pathways in health and disease using an atlas of the underlying anatomy. Front. Neuroinformatics 5.

Yoo, S.W., Guevara, P., Jeong, Y., Yoo, K., Shin, J.S., Mangin, J.-F., Seong, J.-K., 2015. An example-based multi-atlas approach to automatic labeling of white matter tracts. PLoS One 10 (7), e0133337.

Zhang, Y., Brady, M., Smith, S., 2001. Segmentation of brain mr images through a hidden markov random field model and the expectation-maximization algorithm. IEEE Trans. Med. Imaging 20 (1), 45-57.

Zhang, Y., Zhang, J., Oishi, K., Faria, A.V., Jiang, H., Li, X., Akhter, K., Rosa-Neto, P., Pike, G.B., Evans, A., et al., 2010. Atlas-guided tract reconstruction for automated and comprehensive examination of the white matter anatomy. Neuroimage 52 (4), 1289-1301. 